九州大学学術情報リポジトリ

Kyushu University Institutional Repository

\title{
A new magnetosphere-ionosphere coupling scheme for temporal and global magnetospheric MHD simulations
}

Yoshikawa, Akimasa

Department of Earth and Planetary Sciences, Kyushu University | Space Environment Research Center, Kyushu University

Nakata, Hiroyuki

Graduate school of Sciences and Technology, Chiba University

Nakamizo, Ao i

CREST, Japan Science and Technology Agency | Department of Earth and Planetary Sciences, Kyushu University

Uozumi, Te i j i

Space Environment Research Center, Kyushu University

他

https://doi.org/10.5109/13529

出版情報: 九州大学大学院理学研究院紀要 : Series D, Earth and planetary sciences. 32 (2)， pp. 87-94, 2009-03-02. Faculty of Science, Kyushu University

バージョン :

権利関係 : 
Mem. Fac. Sci., Kyushu Univ., Ser. D, Earth \& Planet. Sci., Vol. XXXII, No. 2, pp. 87-94, March 2, 2009

\title{
A new magnetosphere-ionosphere coupling scheme for temporal and global magnetospheric MHD simulations
}

\author{
Akimasa Yoshikawa ${ }^{*}, * *$, Hiroyuki Nakata ${ }^{* * *}$, Aoi Nakamizo ,**** \\ Teiji Uozumi $^{* *}$, Masahiro Itonaga ${ }^{* * * * *}$ and Kiyohumi Yumoto, ${ }^{* * *}$
}

\begin{abstract}
We describe a new process of the magnetosphere-ionosphere (MI) coupling for global MHD simulations. In the MI coupling process, field-aligned currents and electric potentials interact with each other in the region between the ionosphere and the inner boundary of the magnetosphere. In order to examine these field-aligned currents and electric potentials selfconsistently, we consider the boundary condition as a problem of wave reflection, assuming that the changing of field-aligned currents as a result of MI-coupling process is associated with the reflection of shear Alfven waves. Separating the perturbed components from the correct solutions, the equation of current continuity of these components is considered. Then we determine the perturbed components generated by the MI coupling. Since the perturbed components of the electric potentials are associated with the reflected shear Alfven wave, we can describe a spatiotemporal development of ionospheric potential as summation of reflection processes of shear Alfven wave at the inner boundary, and then the new process is applicable to the general problems of the MI coupling.
\end{abstract}

Keywords: MI-coupling, Alfvenic-FAC, Global MHD-simulation

\section{Introduction}

Global Magneto-Hydro-Dynamics (MHD) simulation is the powerful tool for studying the magnetospheric dynamics affected by the solar wind. In the global MHD simulations, the coupling between magnetosphere and ionosphere is also an important factor because the magnetosphere and the ionosphere are connected by Field-Aligned Currents (FACs) that carry energy and momentum. In order to calculate the variations of the magnetosphere correctly, the recent global MHD simulations (e.g., Tanaka 1995, Raeder et al., 2001; Lyon et al., 2004) have managed to include the process of the Magnetosphere-Ionosphere (MI) coupling expressed by a current closure condition between FACs and ionospheric current and tangential continuity condition of electric filed across the ionospheric boundary,

Manuscript received on 10 September 2008; accepted on 8 December 2008

* Department of Earth and Planetary Sciences, Kyushu University, Fukuoka, 812-8581, JAPAN

** Space Environment Research Center, Kyushu University, Fukuoka, 812-8581, JAPAN; yoshi@geo.kyushu-u.ac.jp

*** Graduate school of Sciences and Technology, Chiba University, Chiba, 263-8522, JAPAN

**** CREST, Japan Science and Technology Agency, Kawaguchi, 32-0012, JAPAN

***** Faculty of Education, Yamaguchi University, Yamaguchi, 753-8511, JAPAN 


$$
\begin{gathered}
\nabla_{\perp}^{\left(r_{I}\right)} \cdot\left(\stackrel{\leftrightarrow}{\Sigma} \mathbf{E}^{\mathrm{I}}\right)=-j_{/ /}^{\mathrm{I}}, \\
\mathbf{E}^{\mathrm{I}}=\mathbf{E}^{\mathrm{M}\left(r_{I}\right)},
\end{gathered}
$$

where $j_{/ /}^{\mathrm{I}}$ is the $\mathrm{FAC}$ at the ionospheric plane, $\ddot{\Sigma}$ is a field-aligned-integrated ionospheric conductivity tensor, $\mathbf{E}^{\mathrm{I}}=-\nabla \Phi^{\mathrm{I}}$ is an ionospheric electric field described by an electrostatic potential $\Phi^{\mathrm{I}}, \mathbf{E}^{\mathrm{M}\left(r_{I}\right)}$ is a magnetospheric electric field mapping just on the ionosphere. Here, notations $/ /$ and $\perp$ denote the quantities in the parallel and perpendicular directions to the background geomagnetic filed $\mathbf{B}_{\mathbf{0}}$ while, superscript $\left(\mathrm{r}_{\mathrm{I}}\right)$ means operation at ionospheric altitude $r=r_{I}$. In general, magnetospheric MHD simulator produces velocity field $\mathbf{v}^{\mathrm{MHD}}$ and magnetic field $\mathbf{b}^{\mathrm{MHD}}$ according to their dynamics. The FACs of MHD fields can be described by shear of $\mathbf{b}^{\mathrm{MHD}}$ along $\mathbf{B}_{0}$ as

$$
j_{/ /}^{\mathrm{MHD}}=\hat{e}_{B} \cdot\left(\nabla_{\perp}^{\left(r_{M}\right)} \times \mathbf{b}^{\mathrm{MHD}}\right),
$$

which is connected to $j_{/ /}^{\mathrm{I}}$ by a relation $j_{/ /}^{\mathrm{I}}=\alpha j_{/ /}^{\mathrm{MHD}}$, where $\alpha$ is a geometrical scale factor when an inner boundary of MHD region $\left(\mathrm{r}=\mathrm{r}_{M}\right)$ and ionospheric plane $\left(\mathrm{r}=\mathrm{r}_{I}\right)$ cannot be conformed. At the inner boundary of MHD region, we can determine the magnetospheric potential $\Phi^{\mathrm{MHD}}$, from the following Poisson equation

$$
\nabla_{\perp}^{\left(r_{M}\right) 2} \Phi^{\mathrm{MHD}}=\nabla_{\perp}^{\left(r_{M}\right)} \cdot\left(\mathbf{v}_{M H D} \times \mathbf{B}_{0}\right)
$$

where $\hat{e}_{B}=\mathbf{B}_{0} / B_{0}$ is a unit vector along $\mathbf{B}_{0}$. If there are no potential drop between inner boundary of MHD region and ionosphere along $\mathbf{B}_{0}, \mathbf{E}^{\mathrm{M}\left(r_{I}\right)}$ is simply described by

$$
\mathbf{E}^{\mathrm{M}\left(r_{I}\right)}=-\nabla_{\perp}^{\left(r_{I}\right)} \Phi^{\mathrm{MHD}} .
$$

In nature, both the magnetospheric potential and the FACs need to be simultaneously satisfied the relationship between (1) to (5) and the system is always in a harmonious MI-coupled states. If we can describe the MI-coupling process using first-principle simulation, this harmonious state is naturally achieved at any time, however, if we need to separately solve magnetospheric dynamics and ionospheric conditions, a solution for coupled states is inevitably required.

There are some types of solutions of (1) and (2) for adopting boundary condition of the inner magnetosphere. Most of the previous studies had managed the following solution. First, FACs are determined by magnetic perturbations at the inner boundary of the magnetosphere. Mapping these FACs into the ionosphere along $\mathbf{B}_{0}$, the ionospheric electrostatic potentials are calculated from (1) and the resultant potentials are mapped out to the inner boundary of the magnetosphere with the following sequentce

$$
j_{/ /}^{\mathrm{MHD}} \rightarrow j_{/ /}^{\mathrm{I}} \text { and then } \Phi^{\mathrm{I}} \rightarrow \Phi^{\mathrm{MHD}} .
$$

On the other hand, Janhunen (1998) had proposed another type of solution that the electric potentials determined by velocity fields through (4) at the inner boundary of MHD region were mapped into the ionosphere and then the FACs were calculated from the electric potentials using (1). The resultant FACs are mapped out to the inner boundary and then transformed into magnetic field perturbation with the following sequence

$$
\Phi^{\mathrm{MHD}} \rightarrow \Phi^{\mathrm{I}} \quad \text { and then } \quad j_{/ /}^{\mathrm{I}} \rightarrow j_{/ /}^{\mathrm{MHD}} .
$$


For convenience, we named the former procedure as "potential-generator method" and the latter procedure as "FAC-generator method". Furthermore, Kan and Sun (1996) used another type of solution for (1) to investigate development of ionospheric current system during substorm expansion phase. They assumed that the ionosphere received both of FACs and electric field of incident shear Alfven wave and that produced reflected shear Alfven wave. We named this procedure as "KS method".

In case of steady states, the potential-generator method and the FAC-generator method should give the identical solution for MI-coupled system. However, as for the dynamical system, we cannot expect to find the identical MI-coupled harmonious states from these two methods because these boundary conditions do not consider the simultaneous continuities of both potentials (convection) and FACs (magnetic shear) between ionosphere and inner boundary. As for the KS method, we cannot expect to produce correct reflection field either. Because in the KS-method, the FAC of shear Alfven wave is closed to the total irrotational part of ionospheric current including background current. However, role of shear Alfven wave in the MI-coupled system is mediating accumulated polarization charge caused by change of torsional force balance between Ampere force $(\mathbf{J x B})$ and pressure gradient force $(-\nabla \mathrm{P})$ along $\mathbf{B}_{\mathbf{0}}$ and not describing total amount of FACs itself.

Clearly, shear Alfven wave carries torsional components of disturbed MHD fields to the inner boundary and brings associated potential and FACs disturbances to the ionosphere, which should become driving source for reflected fields of ionospheric origin. As discussed by Yoshikawa et al. (2002), to calculate the correct reflection field from the ionosphere, the total FAC of shear Alfven waves flowing into the ionosphere composed by incident and reflected components are need to be balanced to the divergence part of ionospheric current excited by the incident and reflected (secondary polarized) electric field. To correctly simulate the MI-couple system, we need to develop a new coupling algorithm that can describe harmonious state even in a time-development system, and their changing is mediated by the shear Alfvenic disturbances.

In this paper, we describe a new process of the MI-coupling, which suit to time-development regime: both electrostatic potential and FACs at magnetospheric inner boundary are simultaneously mapped on the ionosphere, and then the reflection components including the Alfvenic-FACs are generated to totally satisfy the MI-coupling relation with the following sequence

$$
\Phi^{\mathrm{MHD}} \rightarrow \Phi^{\mathrm{I}}, j_{/ /}^{\mathrm{MHD}} \rightarrow j_{/ /}^{\mathrm{I}} \text { and then } \Phi^{\mathrm{I}} \rightarrow \Phi^{\mathrm{MHD}}, j_{/ /}^{\mathrm{I}} \rightarrow j_{/ /}^{\mathrm{MHD}} .
$$

We named this procedure as "Alfvenic-coupling algorithm".

In Section 2, we will discuss about the detail of Alfvenic coupling algorithm and derive a new formulation for current closure condition between FACs and ionospheric current. This formulation explicitly shows that time-development of MI-coupling process is caused by excitation of shear Alfvenic disturbances from the ionosphere if any differences between magnetospheric and ionospheric convections at their interface has caused by any of disturbances. Furthermore, we will clarify the context that the MI-coupled variables at the present time is as a result of long succession of reflection and mode conversion process of MHD disturbances at the ionosphere. In other words, progress of MI-coupling system is described by the reflection and mode conversion process of MHD waves at the ionosphere. In Section 3, we will discuss about the differences between the potential-generator model and the FACgenerator model form viewpoint of progress of MI-coupling system, and conclude that the potentialgenerator model would gives more preferable results than the FAC-generator model. However, it is clarified that the potential generator model also cannot give a correct reflected field in a temporal MIcoupling process. Because, in reality, that perturbation components of ionospheric potential acts as not only the mere polarization field but the inductive field of Alfvenic-FACs, which assures the conservation law of energy and momentum in the temporal MI-coupling. 


\section{Alfvenic-Coupling Algorithm}

Concept of Alfvenic-Coupling in the electrostatic MI-coupling process proposed in this paper is as follows. When a distribution of ionospheric current is changed from steady state by mapping of magnetospheric disturbances and/or conductivity changes, polarization potential " $\delta \mathrm{S}$ " is instantaneously generated to preserve the current continuity conditions. This polarization potential is projected as feed back component to the inner boundary of MHD region, which excites the Alfvenic-FACs. Therefore, current continuity condition has to be included this radiant Alfvenic-FACs. In this sense, $\delta \mathrm{S}$ is not only polarization electric field for ionospheric current continuity but also an inductive field of Alfvenic disturbances.

\subsection{Separation of MI-coupled Solution into Primary and Secondary Variables}

To apply the process of Alfvenic-Coupling for numerical simulations, we separate true solution (harmonious state) of $\left(\mathbf{E}^{\mathrm{I}}, j^{\mathrm{I}} / /\right)$ and $\left(\mathbf{v}^{\mathrm{MHD}}, \mathbf{b}^{\mathrm{MHD}}\right)$, which simultaneously satisfy MI-coupled relation and MHD dynamics, into primary and secondary terms in a discrete time-domain as

$$
\begin{gathered}
\mathbf{E}_{k}^{\mathrm{I}}=\mathbf{E}_{\mathrm{p}, k}-\nabla_{\perp} \delta S_{k}^{\uparrow} \\
j_{/ /, k}^{\mathrm{I}}=j_{/ / \mathrm{p}, k}+\delta j_{/ /, k}^{\uparrow}, \\
\mathbf{v}_{k}^{\mathrm{MHD}}=\mathbf{v}_{\mathrm{p}, k}^{\mathrm{MHD}}+\delta \mathbf{v}_{k}^{\mathrm{MHD}} \\
\mathbf{b}_{k}^{\mathrm{MHD}}=\mathbf{b}_{\mathrm{p}, k}^{\mathrm{MHD}}+\delta \mathbf{b}_{k}^{\mathrm{MHD}},
\end{gathered}
$$

where subscript $\mathrm{p}$ means primary terms determined only from the magnetospheric condition before interacting with the ionosphere, while symbol $\delta$ indicates secondary term generated as a result of MIcoupling process satisfying (1) and (2). The subscript $k$ denotes physical quantities at discrete time step $t=t_{\mathrm{k}}(k=0,1,2,3 \cdots)$. In this model, we simply assume that the region between inner MHD boundary and ionosphere is connected through the electrically equipotential geomagnetic field line and the FACs are flowing therein with no vertical divergence conditions. Therefore, total electrostatic potential and the FACs satisfies (1) and (2) at $t=t_{\mathrm{k}}$ are given by

$$
\begin{gathered}
\Phi_{k}^{\mathrm{MHD}}=\Phi_{k}^{\mathrm{I}}=\Phi_{p, k}^{\mathrm{MHD}}+\delta S_{k}^{\uparrow} \\
j_{/ /, k}^{\mathrm{MHD}}=\alpha\left(j_{/ / \mathrm{p}, k}+\delta j_{/ /, k}^{\uparrow}\right) .
\end{gathered}
$$

Variable $_{\mathrm{S}} \Phi_{\mathrm{p}, k}^{\mathrm{MHD}}, \quad j_{/ / \mathrm{p}, k}$, and $\mathbf{E}_{p, k}=-\nabla_{\perp}^{\left(r_{I}\right)} \Phi_{\mathrm{p}, k}^{\mathrm{MHD}}$ can be obtained from MHD fields before interacting with ionosphere at time step $t=t_{\mathrm{k}}$ as

$$
\begin{aligned}
& \nabla_{\perp}^{\left(r_{M}\right) 2} \Phi_{\mathrm{p}, k}^{\mathrm{MHD}}=\nabla_{\perp}^{\left(r_{M}\right)} \cdot\left(\mathbf{v}_{\mathrm{p}, k}^{\mathrm{MHD}} \times \mathbf{B}_{0}\right), \\
& j_{/ / \mathrm{p}, k}=\alpha^{-1} \mu_{0}^{-1} \hat{e}_{B} \cdot\left(\nabla_{\perp}^{\left(r_{M}\right)} \times \mathbf{b}_{\mathrm{p}, k}^{\mathrm{MHD}}\right) .
\end{aligned}
$$

If the system is in the steady or harmonious states, there are no reflected disturbances from the ionosphere.

\subsection{Renormalization of Radiant Alfvenic-FAC to the Current Closure Condition}

Variable of $\delta S_{k}^{\uparrow}$ is an electrostatic potential for polarization fields, which are instantaneously generated if the primary variables $\left(\mathbf{E}_{\mathrm{p}, k}, j_{/ / \mathrm{p}, k}\right)$ cannot satisfy the current closure condition of (1) in their own. The newly generated part of FACs at the inner boundary: $\delta j_{l, k}^{\mathrm{MHD} \uparrow}=\alpha^{-1} \delta j_{l, k}^{\uparrow}$ should satisfy 


$$
\delta j_{l /, k}^{\mathrm{MHD} \uparrow}=\mu_{0} \hat{e}_{B} \cdot\left(\nabla^{\left(r_{M}\right)} \times \delta \mathbf{b}_{k}^{\mathrm{MHD}}\right) .
$$

Using Faraday's law with (17), we have

$$
\frac{\partial}{\partial t} \delta j_{/ /, k}^{\mathrm{MHD} \uparrow}=-\mu_{0} \nabla_{/ /} \nabla_{\perp}^{2} \delta S_{k}^{\uparrow} .
$$

Since $\delta S_{k}^{\uparrow}$ is generated during time scale of $\Delta t_{\mathrm{k}}=t_{\mathrm{k}}-t_{\mathrm{k}-1},(18)$ can be written in the form of

$$
\delta j_{l /, k}^{\mathrm{MHD} \uparrow}=\tilde{L}_{A} \nabla_{\perp}^{2} \delta S_{k}^{\uparrow} \equiv-\Sigma_{A, k} \nabla_{\perp}^{2} \delta S_{k}^{\uparrow} .
$$

The operator $\tilde{L}_{A}=\mu_{0} \Delta t_{k} \nabla_{/ /}$extracts a value of $\mu_{0}^{-1} \times$ (inverse of propagation speed of disturbances in the direction of geomagnetic field lines) when it acts on any disturbances and which gives the FAC with $-\nabla_{\perp}^{2} \delta S_{k}^{\uparrow}$. Since $-\nabla_{\perp}^{2} \delta S_{k}^{\uparrow}$ represents eigenstate of Alfvenic disturbances in the MHD medium, $\tilde{L}_{A}$ can be regarded as an operator extracting Alfvenic conductance: $\sum_{\mathrm{A}, \mathrm{k}}$ as eigenvalue of $-\nabla_{\perp}^{2} \delta S_{k}^{\uparrow}$. In the case of MHD, we had already known that $\sum_{\mathrm{A}, \mathrm{k}}$ is given by

$$
\Sigma_{A, k}=\left(\mu_{0} V_{A}\right)^{-1}
$$

(e.g., Kan and Sun 1996), where $V_{\mathrm{A}}$ is the Alfven velocity at the inner boundary of MHD regions. Application of formula (19) to the global simulation would be validated if grid-spaces of MHD simulation were enough fine regarding background magnetic field and plasma density as uniform.

Using (9) to (20) with (1), we finally obtain a determination equation for $\delta S_{k}^{\uparrow}$ as

$$
-\nabla_{\perp}^{\left(r_{I}\right)} \cdot \vec{\Sigma}_{k} \nabla_{\perp}^{\left(r_{I}\right)}\left(\Phi_{\mathrm{p}, k}^{\mathrm{MHD}}+\delta S_{k}^{\uparrow}\right)=\alpha\left(j_{/ / \mathrm{p}, k}-\Sigma_{A, k} \nabla_{\perp}^{2\left(r_{M}\right)} \delta S_{k}^{\uparrow}\right)
$$

In this formulation, $\mathbf{b}_{\mathrm{MHD}, \mathrm{p}, \mathrm{k}}$ and $\mathbf{v}_{\mathrm{MHD}, \mathrm{p}, \mathrm{k}}$ become inputs for MI-coupling relation and output is the polarization electrostatic potential $\delta S_{k}^{\uparrow}$ in the ionosphere. Using frozen-in condition for MHD fields with (17) and (19), we have the following magnetospheric disturbances at inner boundary of MHD region as a result of MI-coupling process at $t=t_{\mathrm{k}}$

$$
\begin{gathered}
\delta \mathbf{v}_{k}^{\mathrm{MHD}}=\frac{-\nabla \delta S_{k}^{\uparrow} \times \hat{e}_{B}}{B_{0}} \\
\delta \mathbf{b}_{k}^{\mathrm{MHD}}=\frac{\nabla_{\perp} \delta S_{k}^{\uparrow} \times \hat{e}_{B}}{V_{A}}=-\sqrt{\mu_{0} \rho_{0}} \delta \mathbf{v}_{k}^{\uparrow} .
\end{gathered}
$$

By adding these disturbances to the inner boundary of MHD region, the MI-coupling process at $t=t_{\mathrm{k}}$ is then completed.

As done by many previous studies, if we set the total ionospheric potential, $\Phi_{k}^{\mathrm{I}}$ to be explicit variables in the MI-coupling relation, (21) can be rewritten to derive equation for $\Phi_{k}^{\mathrm{I}}$ as

$$
-\nabla_{\perp}^{\left(r_{I}\right)} \cdot\left(\vec{\Sigma}_{k} \nabla_{\perp}^{\left(r_{I}\right)} \Phi_{k}^{\mathrm{I}}\right)=-\alpha\left[j_{/ / \mathrm{p}, k}-\Sigma_{A, k} \nabla_{\perp}^{\left(r_{M}\right)} \cdot\left(\nabla_{\perp}^{\left(r_{M}\right)} \Phi_{k}^{\mathrm{I}}-\mathbf{v}_{\mathrm{p}, k}^{\mathrm{MHD}} \times \mathbf{B}_{0}\right)\right]
$$

The last term of the right-hand side of (24) is an additional term in many of the previous studies. Renormalization of reflected Alfvenic-FACs into the current closure condition make possible disturbed ionospheric potential to act as not only the mere polarization field for current continuity but the inductive field of shear Alfven mode, which assures the conservation law of energy and momentum in the temporal MI-coupling (Yoshikawa et al., 2002). This formulation explicitly shows that time-development of MIcoupling process is caused by excitation of shear Alfven wave from the ionosphere, if the difference 
between magnetospheric and ionospheric convections at their interface has caused by any of disturbances.

\subsection{Separation of MI-coupling process into harmonious state and reflection processes}

The primary MHD variables at $t=t_{\mathrm{k}}$ before interacting with the ionosphere should be written in the form of

$$
\begin{gathered}
j_{/ / \mathrm{p}, k}^{\mathrm{MHD}}=\alpha^{-1} j_{/ /, k-1}^{\mathrm{I}}+\delta j_{/ /, k}^{\downarrow}, \\
\Phi_{\mathrm{p}, k}^{\mathrm{MHD}}=\Phi_{k-1}^{\mathrm{I}}+\delta S_{k}^{\downarrow},
\end{gathered}
$$

where $\left(\delta j_{l, k}^{\downarrow}, \delta S_{k}^{\downarrow}\right)$ are the MHD disturbances newly reached on the inner boundary of MHD region from the upper part of magnetosphere at $t=t_{k}$. Similarly, ionospheric variables can be separated as

$$
\begin{gathered}
\Phi_{k}^{\mathrm{I}}=\Phi_{k-1}^{\mathrm{I}}+\delta S_{k}^{\downarrow}+\delta S_{k}^{\uparrow} \\
\vec{\Sigma}_{k}=\vec{\Sigma}_{k-1}+\delta \vec{\Sigma}_{k}
\end{gathered}
$$

where $\delta \Sigma_{k}$ is the newly generated part of ionospheric conductivity fluctuation at $t=t_{\mathrm{k}}$. Using Eqs. (25) and (26), the total FAC on the ionosphere and ionospheric potential at $t=t_{\mathrm{k}}$ can be represented by

$$
\begin{aligned}
j_{l, k}^{\mathrm{I}} & =j_{l, k-1}^{\mathrm{I}}+\alpha\left(\delta j_{l, k}^{\downarrow}+\delta j_{l, k}^{\uparrow}\right) \\
& =j_{l, k=0}^{\mathrm{I}}+\alpha \sum_{i=1}^{k}\left(\delta j_{\|,, i}^{\downarrow}+\delta j_{\|,, i}^{\uparrow}\right) \\
\Phi_{k}^{\mathrm{I}} & =\Phi_{k-1}^{\mathrm{I}}+\delta S_{k}^{\downarrow}+\delta S_{k}^{\uparrow} \\
& =\Phi_{k=0}^{\mathrm{I}}+\sum_{i=1}^{k}\left(\delta S_{i}^{\downarrow}+\delta S_{i}^{\uparrow}\right)
\end{aligned}
$$

respectively, where $k=0$ means an initial condition of MI-coupled system at $t=t_{0}$. Using (27) and (28), (21) can be separated into the current closure condition at $t=t_{\mathrm{k}-1}$ and reflection and mode conversion process of MHD disturbances at $t=t_{\mathrm{k}}$ as

$$
\begin{gathered}
\nabla_{\perp}^{\left(r_{I}\right)} \cdot \vec{\Sigma}_{k-1} \mathbf{E}_{k-1}^{\mathrm{I}}=-j_{l, k-1}^{\mathrm{I}}, \\
-\nabla_{\perp}^{\left(r_{I}\right)} \cdot\left(\vec{\Sigma}_{k} \nabla_{\perp}^{\left(r_{I}\right)}\left(\delta S_{k}^{\downarrow}+\delta S_{k}^{\uparrow}\right)\right)+\varepsilon=-\alpha \Sigma_{A, k} \nabla_{\perp}^{\left(r_{M}\right) 2}\left(\delta S_{k}^{\downarrow}-\delta S_{k}^{\uparrow}\right)
\end{gathered}
$$

where

$$
\varepsilon=-\nabla_{\perp}^{\left(r_{I}\right)} \cdot\left(\overrightarrow{\delta \Sigma}_{k} \nabla_{\perp}^{\left(r_{I}\right)} \Phi_{k-1}^{\mathrm{I}}\right)
$$

Here we use a relation $\delta j_{l, k}^{\mathrm{MHD}}=\Sigma_{A, k} \nabla_{\perp}^{\left(r_{M}\right) 2} \delta S_{k}^{\downarrow}$ for representing incident part of FACs at $t=t_{\mathrm{k}}$.

Equation (29) represents harmonious state of MI-coupled system at $t=t_{\mathrm{k}-1}$ in which all of physical variables at the inner boundary of MHD region and on the ionospheric plane satisfy (1) and (2). Clearly, direct source of MI-coupling progress from harmonious state from $t=t_{\mathrm{k}-1}$ to $\mathrm{t}_{\mathrm{k}}$ are incident components of MHD variables or ionospheric conductivity disturbances at $t=t_{\mathrm{k}}$. The term of $\varepsilon$ is a current divergence originating ionospheric conductivity fluctuation, which corresponds to a seed of feed back instability as a possible mechanism for explaining excitation and growth of auroral arcs (e.g., Sato 1979), where fluctuations of density and electrostatic field in the ionospheric E-region are coupled with the shear Alfven waves in the magnetosphere. Its energy source is given by a background ionospheric convection. If $\varepsilon=0$, Eq.(30) simply represents an incident and reflection process of shear Alfven modes under inhomogeneous ionospheric conductivity distributions (e.g., Glassmeir, 1983; Itonaga and Kitamura, 1988).

Here we should note that formulation of (27)-(30) shows that the MI-coupling state at $t=t_{\mathrm{k}}$ 
represented by (21) is as a result of long succession of reflection and mode conversion process of MHD disturbances. In other words, progress of MI-coupling system is described by the reflection and mode conversion process of MHD waves at the ionosphere.

\section{Discussion and Conclusions}

It is interesting to consider the question given by Janhunen (1998) "what differences have been including between potential generator model and FAC generator model" from viewpoint of our Alfvenic coupling. If we adopt the context of Alfvenic-coupling algorithm to the potential-generator model, the MI-coupler only receives $j_{/ /, \mathrm{p}, k}^{\mathrm{MHD}}$ and calculates total $\Phi_{k}^{\mathrm{I}}$, therefore the reflection part of FACs is neglected from the right-hand side of (30). On the other hand as for the FAC generator model, the MIcoupler receives $\Phi_{\mathrm{p}, k}^{\mathrm{MHD}}$ and calculates total FACs, therefore, the reflection part of ionospheric potential is neglected from the left-hand side of (30). These differences of solution would give unconformity of reflection fields from the ionosphere. Now let us investigate this unconformity of solutions using simple situation that the ionospheric conductivity distribution is uniform, $\mathbf{B}_{0}$ is vertical to the ionospheric plane, and inner boundary of MHD region directly contact to the ionospheric plane, leads to reduce of (30) to

$$
\Sigma_{P} \nabla_{\perp}\left(\delta S^{\downarrow}+\delta S^{\uparrow}\right)=\Sigma_{A} \nabla_{\perp}\left(\delta S^{\downarrow}-\delta S^{\uparrow}\right) .
$$

This relation gives well-known reflection coefficient of shear Alfven wave: $R_{A}=\left(\Sigma_{A}-\Sigma_{P}\right) /\left(\Sigma_{A}+\Sigma_{P}\right)$, where $R_{A} \equiv \delta S^{\downarrow} / \delta S^{\uparrow}$ (e.g., Scholer 1970), hence, we can say that Alfvenic-coupling model involves correct reflection process of shear Alfven wave. On the other hand, in the potential generato $\mathrm{r}$ model, corresponding temporal MI-couple process is described by $\Sigma_{P} \nabla_{\perp}\left(\delta S^{\downarrow}+\delta S^{\uparrow}\right)=\Sigma_{A} \nabla_{\perp} \delta S^{\downarrow}$ and reflection coefficient become $R_{A}^{\mathrm{pol}}=\left(\Sigma_{A}-\Sigma_{P}\right) / \Sigma_{P}$. While, in the FAC generator model, MI-couple process is described by $\Sigma_{P} \nabla_{\perp} \delta S^{\downarrow}=\Sigma_{A} \nabla_{\perp}\left(\delta S^{\downarrow}-\delta S^{\uparrow}\right)$ and reflection coefficient become $R_{A}^{\mathrm{FAC}}=\left(\Sigma_{A}-\Sigma_{P}\right) / \Sigma_{A}$. For typical condition of $\Sigma_{\mathrm{A}} \sim 1 \mathrm{mho}$ and $\Sigma_{\mathrm{P}} \sim 10 \mathrm{mho}$, reflection coefficient in the case of Alfvenic-coupling, potential-generator, and FAC-generator models become $-0.82,-0.9$, and -8.0 , respectively. We can understand that reflection field given by the FAC-generator model is far from the correct solutions.

A ratio of MI-coupled components given by the potential generator model to by the Alfvenic coupling model in the reflection coefficient: $R_{\mathrm{A}}$, the ionospheric potential: $\delta \Phi^{\mathrm{I}}=\left(1+R_{A}\right) \delta S^{\downarrow}$, and the FACs flowing into the ionosphere: $\delta j_{/ /}^{1}=\Sigma_{A} \nabla_{\perp}^{2}\left(\delta S^{\downarrow}-\delta S^{\uparrow}\right)$ become $1+, \alpha_{P}^{-1},\left(1+\alpha_{P}^{-1}\right) / 2$, and $1-\alpha_{P}^{-1} / 2$, respectively, where $\alpha p=\sum p / \sum_{A}$. In a case of high-conducting ionosphere $\left(\alpha_{p}>>1\right)$ i.e., realizing in the dayside and/or auroral ionosphere, the potential-generator model gives almost the same values of $R_{\mathrm{A}}$ and $\delta j_{/ /}^{\mathrm{I}}$ given by Alfvenic-coupling model, while, the potential-generator model gives $50 \%$ underestimated values of $\delta \Phi^{\mathrm{I}}$ compared with that of Alfvenic-coupling model. On the other hand, in the case of lowconducting ionospheric condition such as night-side and no-particle precipitation regions $\left(\alpha_{p} \sim 1\right)$, the potential-generator model gives almost the same values of $\delta \Phi^{\mathrm{I}}$ compared with that of Alfvenic-coupling model. While, $R_{\mathrm{A}}$ and $\delta j_{/ /}^{\mathrm{I}}$ in the potential-generator model become $100 \%$ overestimated and $50 \%$ underestimated values, comparing with that of Alfvenic-coupling model, respectively.

We can conclude that from viewpoint of progress of MI-coupling system, the potential-generator model would give more preferable results than the FAC-generator model. The physical reason for this difference come from quite small conductance of Alfvenic-FACs compared with conducatance of ionosphere. In the potentials-generator model, reflected Alfvenic-FACs are neglected from the temporal MI-coupled process, while in the FAC-generator model, ionospheric current generated by the reflected fields are neglected from the system, and thus, discrepancy from true solution of the Alfvenic- coupling algorithm become larger for the FAC-generator model than the potential-generator model. However, it 
is clear that the potential generator model also cannot give a correct reflected field in a temporal MIcoupling process because, in reality, perturbation components of ionospheric potential acts as not only the mere polarization field but the inductive field of Alfvenic-FACs which assures the conservation law of energy and momentum in the temporal MI-coupling (Yoshikawa et al., 2002). It is desirable that this process is adopted not only by the boundary condition of global MHD simulations but by general problems of the magnetosphere-ionosphere coupling or the other ionospheric models. Of course, this process should be evaluated by comparing with the results determined by the previous boundary conditions. Currently we are preparing for the global MHD simulation with the present condition, modifying the TVD scheme developed by Tanaka (1995). In the near future, we will show the results of the global MHD simulation using the new process presented here.

\section{Acknowledgements}

We thank an anonymous reviewer who provided constructive criticisms which improved the quality of this paper.

\section{References}

Lyon, J.G., Fedder, J.A., and Mobarry, C.M. (2004) The Lyon-Fedder Mobarry (LFM) global MHD magnetospheric simulation code, J. Atmos. Solar Terr., Phys. 66, 1333-1350.

Raeder, J., McPherron, R.L. Frank, L.A., Kokubun, S., Lu, G., Mukai, T., Paterson, W.R., Sigwarth, J.B., Singer, H.J., and Slavin, J.A. (2001) Global simulation of the Geospace Environment Modeling substorm challenge event, J. Geophys. Res., 106, 381-395.

Scholer, M. (1970) On the motion of arti?cial ion clouds in the magnetosphere, Planet. Space Sci., 18, 977-1004.

Tanaka, T. (1995) Generation mechanisms for magnetosphere-ionosphere current systems deduced from a three-dimensional MHD simulation of the solar wind-magnetosphere-ionosphere-coupling processes, J. Geophys. Res., 100, 12057-12074.

Janhunen, P. (1998) On the possibility of using an electromagnetic ionosphere in global MHD simulations, Ann. Geophys., 16, 397-402.

Kan, J.R., and Sun, W. (1996) Substorm expansion phase caused by an intense localized convection imposed on the ionosphere, J. Geophys. Res., 101, 27,271-27,281.

Glassmeier K.H. (1983) Reflection of MHD-waves in the Pc4-5 period range at ionospheres at nonuniform conductivity distributions, Geophys. Res. Lett., 10, 678-681.

Itonaga M., and Kitamura T. (1988) Effect of non-uniform ionospheric conductivity distribution on PC3-5 magneticpulsations - Alfven-wave incidence J. Geomagn. Geoelectr., 40, 1413-1435.

Yoshikawa, A., Yumoto, K., Shinohara, M., and Itonaga, M. (2002) Diarammatic method to describe the self-inductive response of the magnetosphere-ionosphere- atmosphere-Earth electromagnetically coupled system as a quasi-particle excitation, J. Geophys. Res., 107, 1055, doi:10.1029/2000JA000405. 